\title{
The Sky Blue Method as a Screening Test to Detect Misplacement of Percutaneous Endoscopic Gastrostomy Tube at Exchange
}

\author{
Yutaka Suzuki ${ }^{1}$, Mitsuyoshi Urashima ${ }^{2}$, Hideki Yoshida ${ }^{3}$, Tsuyoshi Iwase ${ }^{4}$, Toshiroh Kura ${ }^{5}$, \\ Shin Imazato ${ }^{6}$, Michiaki Kudo ${ }^{7}$, Tomoyuki Ohta ${ }^{8}$, Akihiro Mizuhara ${ }^{9}$, Yutaka Tamamori ${ }^{10}$, \\ Hirohito Muramatsu ${ }^{11}$, Yukio Nishiguchi ${ }^{12}$, Yorihiro Nishiyama ${ }^{13}$, Mikako Takahashi ${ }^{14}$, \\ Shinji Nishiwaki ${ }^{15}$, Masami Matsumoto ${ }^{16}$, Satoshi Goshi ${ }^{17}$, Shigeo Sakamoto ${ }^{18}$, \\ Nobuyuki Uchida $^{19}$, Masashi Ijima ${ }^{20}$, Tetsushi Ogawa ${ }^{21}$, Makoto Shimazaki ${ }^{22}$, \\ Shinichi Takei ${ }^{23}$, Chikou Kimura ${ }^{24}$, Satoyoshi Yamashita ${ }^{25}$, Takao Endo ${ }^{26}$, Masato Nakahori ${ }^{27}$, \\ Akihiko Itoh $^{28}$, Toshiro Kusakabe ${ }^{29}$, Izumi Ishizuka ${ }^{30}$, Takao Iiri ${ }^{31}$, Shingo Fukasawa ${ }^{32}$, \\ Yukitsugu Arimoto ${ }^{33}$, Nobuaki Kajitani ${ }^{34}$, Kazuhiko Ishida ${ }^{35}$, Koji Onishi ${ }^{36}$, Akihiko Taira ${ }^{37}$, \\ Makoto Kobayashi ${ }^{38}$, Yasuto Itano ${ }^{39}$ and Toshiya Kobuke ${ }^{40}$
}

\begin{abstract}
Background During tube exchange for percutaneous endoscopic gastrostomy (PEG), a misplaced tube can cause peritonitis and death. Thus, endoscopic or radiologic observation is required at tube exchange to make sure the tube is placed correctly. However, these procedures cost extensive time and money to perform in all patients at the time of tube exchange. Therefore, we developed the "sky blue method" as a screening test to detect misplacement of the PEG tube during tube exchange.

Methods First, sky blue solution consisting of indigocarmine diluted with saline was injected into the gastric space via the old PEG tube just before the tube exchange. Next, the tube was exchanged using a standard method. Then, we checked whether the sky blue solution could be collected through the new tube or not. Finally, we confirmed correct placement of the tube by endoscopic or radiologic observation for all patients.

Results A total of 961 patients were enrolled. Each tube exchange took 1 to 3 minutes, and there were no adverse effects. Four patients experienced a misplaced tube, all of which were detectable with the sky blue method. Diagnostic parameters of the sky blue method were as follows: sensitivity, 94\% (95\% CI: 92-95\%); specificity, $100 \%$ (95\%CI: $40-100 \%)$; positive predictive value, $100 \%$ (95\%CI: $100-100 \%)$; negative predictive value, $6 \%$ (95\%CI: $2-16 \%$ ).
\end{abstract}

Conclusion These results suggest that the number of endoscopic or radiologic observations to confirm correct replacement of the PEG tube may be reduced to one fifteenth using the sky blue method.

\footnotetext{
${ }^{1}$ Department of Surgery, International University of Health and Welfare, Otawara, ${ }^{2}$ Division of Molecular Epidemiology, The Jikei University School of Medicine, Tokyo, ${ }^{3}$ Yoshida Hospital, Nagaoka, ${ }^{4}$ Kyoto Kujo Hospital, Kyoto, ${ }^{5}$ Naganuma Municipal Hospital, Hokkaido, ${ }^{6}$ PEG Center, Oita Kensei Hospital, Oita, ${ }^{7}$ Onishi Hospital, Fujioka, ${ }^{8}$ Center for Gastroenterology, Sapporo Higashi-Tokushukai Hospital, Sapporo, ${ }^{9} \mathrm{Hi}-$ gashi Washinomiya Hospital, Saitama, ${ }^{10}$ Baba Memorial Hospital, Sakai, ${ }^{11}$ Kiyota Hospital, Sapporo, ${ }^{12}$ Osaka City General Hospital, Osaka, ${ }^{13}$ Nishiyama Clinic, Otsu, ${ }^{14}$ Tsuruoka Kyoritsu Hospital, Tsuruoka, ${ }^{15}$ Department of Internal Medicine, Nishimino Kosei Hospital, Gifu, ${ }^{16}$ Nara Prefectural Gojo Hospital, Gojyo, ${ }^{17}$ Endoscopic Treatment Center, Japan Labour Health and Welfare Organization, Jyoetsu, ${ }^{18}$ Johoku Hospital, Kanazawa, ${ }^{19}$ Haramachi Red-Cross Hospital, Gunma, ${ }^{20}$ Isesaki Municipal Hospital, Isesaki, ${ }^{21}$ Maebashi Red Cross Hospital, Maebashi, ${ }^{22}$ Hirano General Hospital, Gifu, ${ }^{23}$ JA Nigata, Joetsu General Hospital, Jyoetsu, ${ }^{24}$ Kimura Hospital, Sabae, ${ }^{25}$ Social Insurance Shimonoseki Welfare Hospital, Shimonoseki, ${ }^{26}$ Sapporo Shirakaba-dai Hospital, Sapporo, ${ }^{27}$ Digestive Endoscopy Center, Sendai Kousei Hospital, Sendai, ${ }^{28}$ Kusatsu General Hospital, Kusatsu, ${ }^{29}$ Chitose City Hospital, Chitose, ${ }^{30}$ Kohka Public Hospital, Koga, ${ }^{31}$ TACHIKAWA Medical Center, Nagaoka, ${ }^{32}$ Koma Kyoritu Hospital, Minami-alps, ${ }^{33}$ Sunami Syusaikai Hospital, Mihara, ${ }^{34}$ Bizen Cities' Yoshinaga Hospital, Bizen, ${ }^{35}$ Sendai City Medical Center, Sendai, ${ }^{36}$ Matsue Seikyo Genaral Hospital, Matsue, ${ }^{37}$ Tsuyama Chuo Hospital, Tsuyama, ${ }^{38}$ Toyosaka Hospital, Niigata, ${ }^{39}$ Okayama Kyoritsu Hospital, Okayama and ${ }^{40}$ Sunami Syusaikai Hospital, Mihara
}

Received for publication June 17, 2009; Accepted for publication August 3, 2009

Correspondence to Dr. Mitsuyoshi Urashima, urashima@jikei.ac.jp 
Key words: nutritional support, enteral nutrition, complications, endoscopic gastrointestinal surgery, peritonitis

(Inter Med 48: 2077-2081, 2009)

(DOI: 10.2169/internalmedicine.48.2598)

\section{Introduction}

Percutaneous endoscopic gastrostomy (PEG) is the primary choice to establish enteral access for patients unable to take oral feedings (1-3). Although generally considered safe, PEG tube placement and replacement can be associated with potential complications (4). At tube exchange, a misplaced tube can cause peritonitis and death. Thus, endoscopic or radiologic observation is necessary at tube exchange to ensure that the tube is not misplaced. However, extensive time and money are required to perform these procedures for all patients at tube exchange. Therefore, we developed the "sky blue method" as a screening test to detect misplacement of the PEG tube at tube exchange.

\section{Patients and Methods}

\section{Patients}

In this prospective cohort study, patients undergoing PEG tube exchange between January 2007 and December 2008 from 23 hospitals and health care institutions were enrolled. All institutional review boards approved the procedure. Patients who 1) had a PEG inserted within 1 month, 2) had gastrectomy under open surgery, 3) were in poor general condition, 4) and who were allowed semi-solid enteral nutrition were excluded. All patients and their families were informed about possible risks and benefits of performing the sky blue method, and written consent was obtained.

\section{Sky blue method procedure}

One $\mathrm{mL}(4 \mathrm{mg})$ of disodium indigotindisulfonate (Indigocarmine $^{\circledR}$ : Daiichi Sankyo Company, Ltd., Chuou-ku, Tokyo, Japan) was diluted with $100 \mathrm{~mL}$ of saline and injected into the gastric space through the old PEG tube; this solution is referred to as the sky blue solution. Then, the old PEG tube was exchanged with the new PEG tube. The natural back flow of sky blue solution from the gastric space into the new PEG tube was measured. If a natural back flow was not observed, a catheter tip was used to draw in the sky blue solution. Simultaneously, the clinician compressed the patient's abdomen when the flow of indigocarmine solution was evaluated. When more than $10 \mathrm{~mL}$ of the solution was collected, we considered tube placement a success (positive). In contrast, if $\leq 10 \mathrm{~mL}$ of solution was collected, tube placement was considered a failure (negative). Figure 1 shows the procedure of the sky blue method. After the sky blue method, all patients underwent endoscopic or radiologic observation to confirm that the new tube was placed correctly.

\section{Statistical analysis}

All statistical analyses were performed using STATA 8.0 (STATA Corporation, College Station, TX).

\section{Results}

\section{Patient characteristics}

A total of 961 patients were included in this study. Ages ranged from 19 to 106 years (mean, 78.4 12.1 ; median, 81 years). Most patients (59.4\%) were women. Underlying diseases for reduced peroral feeding were as follows: cerebral infarction (45\%); cerebral hemorrhage (17\%), Alzheimer disease $(10 \%)$, Parkinson disease $(6 \%)$, tuberculosis $(0.1 \%)$, cancer $(1.3 \%)$, and others. The tube exchange procedure took 1 to 3 minutes, and there were no adverse effects.

\section{Sky blue method and endoscopic/radiologic obser- vation}

Histogram of aspirated amounts is shown in Fig. 2. Minimum and maximum were $0 \mathrm{~mL}$ and $300 \mathrm{~mL}$, respectively. Mean \pm standard deviation was $43.5 \mathrm{~mL} \pm 30.8 \mathrm{~mL}$, and $25 \% / 50 \% / 75 \%$ was $20 \mathrm{~mL} / 40 \mathrm{~mL} / 65 \mathrm{~mL}$. The amount of aspirated solution was less than $10 \mathrm{~mL}$ in 136 (14\%) patients.

Success and failure judged by the sky blue method and the follow-up endoscopic ( $\mathrm{N}=297)$ or radiologic observation $(\mathrm{N}=664)$ are shown in Table 1 . Four patients had a misplaced tube, all of which were detected with the sky blue method.

A total of 62 patients were judged as failures by the sky blue method, of which 58 were confirmed as successes by endoscopy. On the other hand, 899 were judged as successes by the sky blue method, all of whom were confirmed to be correct via endoscopic or radiologic observation. Although we explored to determine any significant factors among age, gender, underlying diseases, etc using multiple logistic regression models, we could not find the factors affecting false positive cases $(\mathrm{N}=58)$. Moreover, we could not detect the statistical significance in clinical features of 'the sky blue-positive and successfully replaced group' and 'the sky blue-negative but successfully replaced group' by chi-square test or analysis of variance (Table 2).

The diagnostic parameters of the sky blue method were as 

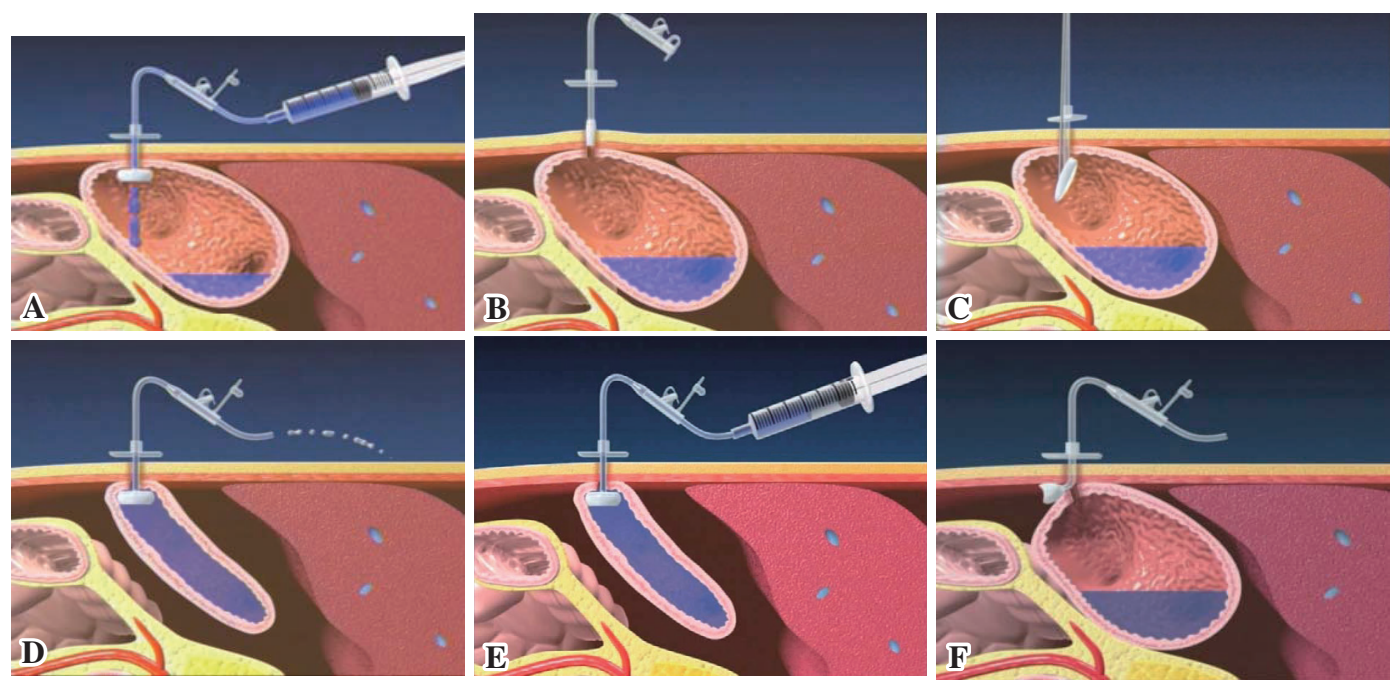

Figure 1. A: One $\mathrm{mL}(4 \mathrm{mg})$ of Indigocarmine ${ }^{\circledR}$ is diluted with $100 \mathrm{~mL}$ of saline and injected into the gastric space through the old PEG tube; this solution is referred to as the sky blue solution. B: The old PEG tube is removed. C: The new PEG tube is inserted. D: The natural back flow of sky blue solution from the gastric space into the new PEG tube is measured. E: If a natural back flow is not observed, a catheter tip was used to draw in the sky blue solution. F: If the new tube is not correctly inserted into the gastric space, the sky blue solution is not drawn in. When more than 10 $\mathrm{mL}$ of the solution was collected, we considered tube placement a success (positive). In contrast, if $\leq$ $10 \mathrm{~mL}$ of solution was collected, tube placement was considered a failure (negative).

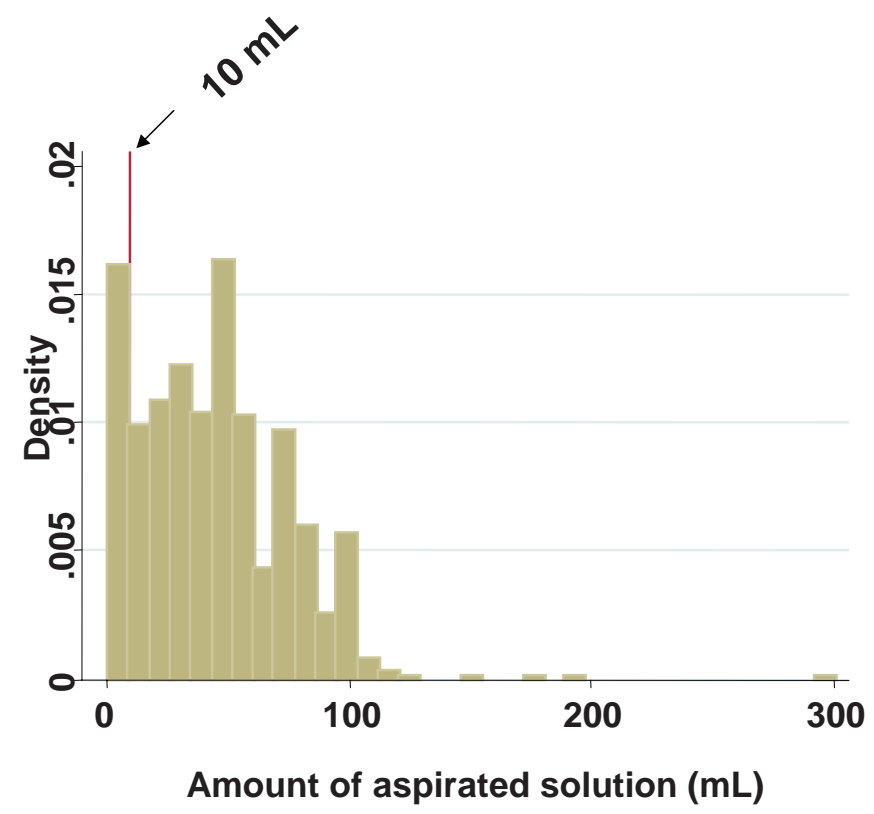

Figure 2. Histogram of aspiration amounts.

Table 1. Results of the Sky Blue Method and Endoscopic Observation to Determine Correct Tube Placement

\begin{tabular}{ccccc}
\hline & & \multicolumn{3}{c}{$\begin{array}{c}\text { Endoscopic observation (Gold Standard) } \\
\text { or radiographic observation }{ }^{* 1}\end{array}$} \\
\hline \multirow{3}{*}{ Sky blue method } & Success & Failure & Total \\
& Fuccess & 899 & 0 & 899 \\
& Failure & 58 & 4 & 62 \\
& Total & 957 & 4 & 961 \\
\hline
\end{tabular}

*1:503 patients were confirmed by radiographic observation 
Table 2. Patients' Characteristics Stratified by Sky Blue Method

\begin{tabular}{|c|c|c|c|c|c|}
\hline \multirow{3}{*}{\multicolumn{2}{|c|}{ variable }} & Total & Sky blue (+) & Sky blue (-) & Sky blue (-) \\
\hline & & & Standard (+) & Standard (+) & Standard (-) \\
\hline & & N: 961 & N: 899 & $\mathrm{~N}: 58$ & $\mathrm{~N}: 4$ \\
\hline \multicolumn{2}{|l|}{ Age } & $78.7 \pm 12.1$ & $78.7 \pm 11.9$ & $78.1 \pm 15.3$ & $80.0 \pm 5.7$ \\
\hline \multicolumn{2}{|l|}{ Male/Female } & $390 / 571$ & $370 / 529$ & $19 / 39$ & $1 / 3$ \\
\hline \multicolumn{6}{|l|}{ Underlying disease } \\
\hline \multicolumn{2}{|c|}{ Cerebral hemorrhage } & 412 & 384 & 26 & 2 \\
\hline \multicolumn{2}{|c|}{ Cerebral infarction } & 153 & 145 & 6 & 2 \\
\hline \multicolumn{2}{|c|}{ Alzheimer disease } & 89 & 85 & 4 & 0 \\
\hline \multicolumn{2}{|c|}{ Parkinson disease } & 54 & 50 & 4 & 0 \\
\hline \multicolumn{2}{|c|}{ Tuberculosis } & 1 & 1 & 0 & 0 \\
\hline \multicolumn{2}{|r|}{ Cancer } & 12 & 12 & 0 & 0 \\
\hline \multicolumn{2}{|c|}{ Poor peroral intake } & 68 & 61 & 7 & 0 \\
\hline \multicolumn{2}{|r|}{ others } & 42 & 161 & 11 & 0 \\
\hline \multicolumn{6}{|l|}{ Time for sky blue } \\
\hline \multirow{3}{*}{$1 \leq$} & $<1 \min$ & 280 & 266 & 10 & 4 \\
\hline & $<5 \min$ & 634 & 591 & 43 & 0 \\
\hline & $5 \min \leq$ & 47 & 42 & 5 & 0 \\
\hline \multicolumn{2}{|l|}{ Years with PEG } & $10.6 \pm 4.8$ & $10.6 \pm 4.8$ & $9.8 \pm 4.3$ & $10.0 \pm 5.4$ \\
\hline \multicolumn{2}{|c|}{$\begin{array}{l}\text { Number of Times of } \\
\text { previous exchange }\end{array}$} & $4.0 \pm 2.4$ & $4.0 \pm 2.5$ & $3.9 \pm 2.3$ & $6.0 \pm 1.4$ \\
\hline
\end{tabular}

follows: sensitivity, $94 \%$ (95\%CI: $92-95 \%)$; specificity, 100\% (95\%CI: 40-100\%); positive predictive value, $100 \%$ (95\%CI: 100-100\%); negative predictive value, 6\% (95\% CI: $2-16 \%)$. Years of experience in performing PEG were not significantly associated with failure of sky blue method (data not shown).

\section{Discussion}

In this study, the sky blue method could detect all 4 tube misplacements. The positive predictive value of the sky blue method was calculated as $100 \%$ and its $95 \% \mathrm{CI}$ was $100 \%$ to $100 \%$. Thus, when tube exchange is considered a success by the sky blue method, patients do not have to undergo endoscopic or radiologic confirmation, although a very minor uncertainty must still be considered. On the other hand, when tube exchange is considered a failure by the sky blue method, patients must undergo endoscopic or radiologic observation to confirm the location of tube. However, by using the sky blue method as a screening test, the number of en- doscopies may be reduced to one fifteenth ( $6 \%$ of patients).

Patients who undergo PEG surgery generally have some underlying diseases and are older, suggesting a higher risk of death. The mortality at $1,3,6$, and 12 months after PEG insertion has been shown to be $16 \%, 20 \%, 25 \%$, and $28 \%$, respectively (5-7). There are few reports showing serious complications and mortality at tube exchange of PEG, although a few cases have been recently reported in Japan. Therefore, if possible, it is better to avoid a surgical procedure, such as endoscopy, in high-risk patients with PEG.

Although this was a prospective cohort study, there are several limitations. The frequency of tube misplacement was relatively rare in this study $(0.4 \%)$. Thus, there is still uncertainty about whether sky blue can detect all misplacements.

In conclusion, the sky blue method may be a safe and simple way to screen for misplacement of the PEG tube at tube exchange. Moreover, the number of endoscopic or radiographic procedures to confirm correct replacement of the PEG tube may be reduced to one fifteenth using the sky blue method.

\section{References}

1. Gauderer MW, Ponsky JL, Izant RJ Jr. Gastrostomy without laparotomy: a percutaneous endoscopic technique. J Pediatr Surg 15: 872-875, 1980.

2. Ponsky JL, Gauderer MW. Percutaneous endoscopic gastrostomy: a nonoperative technique for feeding gastrostomy. Gastrointest Endosc 27: 9-11, 1981.

3. Gopalan $S$, Khanna S. Enteral nutrition delivery technique. Curr Opin Clin Nutr Metab Care 6: 313-317, 2003.
4. Schrag SP, Sharma R, Jaik NP, et al. Complications related to percutaneous endoscopic gastrostomy (PEG) tubes. A comprehensive clinical review. J Gastrointestin Liver Dis 16: 407-418, 2007.

5. Longcroft-Wheaton G, Marden P, Colleypriest B, Gavin D, Taylor G, Farrant M. Understanding why patients die after gastrostomy tube insertion: a retrospective analysis of mortality. JPEN J Parenter Enteral Nutr 33: 375-379, 2009.

6. Johnston SD, Tham TC, Mason M. Death after PEG: results of 
Inter Med 48: 2077-2081, 2009 DOI: 10.2169/internalmedicine.48.2598

the National confidential enquiry into patient outcome and death. Gastrointest Endosc 68: 223-227, 2008.

7. Higaki F, Yokota O, Ohishi M. Factors predictive of survival after percutaneous endoscopic gastrostomy in the elderly: is dementia really a risk factor? Am J Gastroenterol 103: 1011-1016, 2008.

(C) 2009 The Japanese Society of Internal Medicine http://www.naika.or.jp/imindex.html 